\title{
Análisis de sismos interplaca, Pacifíco central de Costa Rica: los sismos de Esterillos (13 de noviembre de 2017) y Herradura (24 de agosto de 2020)
}

\author{
Interplate earthquake analysis, central Pacific of Costa Rica: the Esterillos (November 13, 2017) \\ and Herradura (August 24, 2020) earthquakes
}

\author{
Ronni Quintero-Quintero ${ }^{1}$, Daniela Campos-Durán ${ }^{2, *}$, Hernán Porras-Espinoza ${ }^{3}$, \\ Juan Segura-Torres ${ }^{1}$
}

\begin{abstract}
${ }^{1}$ Observatorio Vulcanológico y Sismológico de Costa Rica, Universidad Nacional. 2386-3000. Universidad Nacional, Heredia, Costa Rica.

${ }^{2}$ Escuela de Ciencias Geográficas, Universidad Nacional. Campus Omar Dengo. Avenida 1, Calle 9. Apartado Postal 86-3000, Heredia, Costa Rica.
\end{abstract}

${ }^{3}$ Sistema Nacional de Monitoreo de Tsunami. Departamento de Física (SINAMOT-OPCOS), Universidad Nacional, Heredia, Costa Rica. -Durán)daniela.campos.duran@una.cr
* Autor para correspondencia: (D. Campos

\section{RESUMEN}

Se analizan dos sismos fuertes que ocurrieron el 13 de noviembre del 2017, 02:28:20

(sismo Esterillos) y el 24 de agosto 2020 a las 21:51:08 (sismo de Herradura) en el Pacífico Central de Costa Rica. El sismo de Herradura se ubicó con latitud $9.501^{\circ} \mathrm{N}$, longitud $84.665^{\circ} \mathrm{W}$, con centroide a $25 \mathrm{~km}$ de profundidad, Mw 5.9 y +1.3 segundos después del tiempo de origen. Este evento se asocia con una falla inversa con dirección noroeste $\left(311^{\circ}\right)$, buzando al noreste en un ángulo bajo de $20^{\circ}$ y con un ángulo de deslizamiento de $98^{\circ}$. El sismo fue reportado sentido desde muy fuerte a fuerte y mantiene estrecha relación con las aceleraciones pico (PGA) registradas instrumentalmente; con las que se determinó que la máxima intensidad en la escala de Mercalli Modificada (MMI) fue de VI, correspondiendo a $12 \% \mathrm{~g}$. El sismo de Esterillos de 2017 se localizó a $14 \mathrm{~km}$ al sureste del sismo de Herradura, con centroide a $21 \mathrm{~km}$ de profundidad, $\mathrm{Mw}$ 6.4. El plano de falla que originó el sismo se orienta en dirección $289^{\circ}$, buzando $17^{\circ}$ al noreste y ángulo de deslizamiento de $85^{\circ}$, con distribución de deslizamientos máximos de $0.6 \mathrm{~m}$, alrededor del centroide y con radio de $\sim 8 \mathrm{~km}$. Estos sismos se ubican en la interfaz sismogénica de las placas de Coco y Caribe (CO-CA) y la mayoría de las réplicas se registraron en los primeros $25 \mathrm{~km}$ de profundidad y con epicentros entre la costa y la trinchera mesoamericana (TMA). Después de ocurrido el sismo de Esterillos, se localizaron sismos corticales en la placa continental y cercanos a la zona epicentral; la deformación transtensional en esta zona se confirma por medio de mecanismos focales obtenidos para algunos de estos sismos y que puede ser indicio de la subducción de montes submarinos en el área.

por pares es responsabilidad Universidad Nacional Autónoma de México.
Palabras clave: Pacífico Central, sismo, localización, mecanismo focal, réplicas.

\section{ABSTRACT}

Two strong earthquakes occurred on November 13, 2017, 02:28:20 (Esterillos Earthquake) and August 24, 2020 at 21:51:08 (Herradura Earthquake) in the Central Pacific of Costa Rica and are analyzed in this work. Herradura earthquake was located at latitude $9.501^{\circ} \mathcal{N}$, longitude $84.665^{\circ} \mathrm{W}$, with centroid at 25 $\mathrm{km}$ depth, Mw 5.9, and +1.3 seconds after the origin time. It is associated with an inverse fault striking northwest $\left(311^{\circ}\right)$, dipping to the northeast at a low angle of $20^{\circ}$ and with a slip angle of $98^{\circ}$. The earthquake was reported felt from strong to very strong; keeping a close relation with the peak accelerations (PGA) registered instrumentally; this data allowed determined that the maximum intensity in the scale of Modified Mercalli (MMI) was of VI, corresponding to $12 \%$ g. Esterillos earthquake, was located 14 $\mathrm{km}$ southeast of the Herradura earthquake, with centroid at $21 \mathrm{~km}$ depth, Mw 6.4. The chosen fault plane where the event originated is striking $289^{\circ}$ to the $\mathcal{N W}$, dipping $17^{\circ}$ to the northeast and with a slip angle of $85^{\circ}$, with circular form slip distribution, reaching maximum of $0.6 \mathrm{~m}$ around the centroid and with a radius of $\sim 8$ $\mathrm{km}$. These earthquakes are located at the seismic interface of the Cocos and Caribbean plates (COCA) and most of the aftershocks were recorded in the first $25 \mathrm{~km}$ depth and with epicenters between the coast and the Middle America Trench (MAT). Cortical Caribbean events were located after the Esterillos event and close to it epicenter, the transtensional deformation in the continental Esterillos epicentral area and confirmed with calculated focal mechanism for some of these events could be related to subducted seamount in the zone.

Keywords: Central Pacific, earthquake, location, focal mechanism, aftershocks. 


\section{Introducción}

El 24 de agosto de 2020 a las 21:51:08 (hora UTC), se presentó un evento sísmico, registrado por la red sísmica del Observatorio Vulcanológico y Sismológico de Costa Rica de la Universidad Nacional (OVSICORI-UNA), $13 \mathrm{~km}$ al sur de la ciudad de Jacó, Puntarenas, Pacífico Central de Costa Rica, con magnitud de 6.3 Mwp (Tsuboi et al., 1995), magnitud Richter Ml 6.1 y a una profundidad de $18 \mathrm{~km}$. Con base en los reportes de la población en la página oficial de Facebook del OVSICORI-UNA, se determinó que el sismo fue sentido desde muy fuerte a fuerte en gran parte del territorio nacional. Fue reportado como percibido en forma oscilatoria y mecida, las ondas sísmicas fueron registradas por los instrumentos de baja ganancia instalados en distintos puntos del país y que mostraron la aceleración máxima del suelo en las componentes horizontales; no sobrepasó 15\% del valor de la gravedad.

En este trabajo se llevó a cabo la relocalización del sismo principal, así como el cálculo de la inversión de parámetros de la fuente sísmica, como el centroide, ubicación y tiempo de origen, mecanismo focal, plano de falla (principal y auxiliar), distribución de las réplicas, distribución de deslizamientos en la falla, cambio de esfuerzos estáticos de Coulomb y el análisis del contexto sismotectónico donde se localizó el sismo. Además, con base en las aceleraciones pico (PGA) registradas en estaciones sísmicas, se determinaron las intensidades instrumentales y su correspondencia en la escala de Intensidad Mercalli Modificada (IMM), lo que permitió generar un mapa de intensidades para todo el país, el valor máximo registrado en la escala IMM fue de VI. Esto se comparó con los reportes de la población en la red social Facebook del OVSICORI-UNA, en su mayoría el sismo fue reportado desde muy fuerte a fuerte, además se reportó la caída de objetos en áreas pobladas cercanas al epicentro (13 km), lo que coincide con una intensidad máxima IMM de VI.

Aunque la idea principal en este trabajo fue analizar el sismo de Herradura del 2020 y teniendo que en el 2017 se localizó un sismo, con magnitud Mw 6.4 (llamado sismo de Esterillos en este trabajo) y muy cercano al sismo de Herradura es que también procedemos a la caracterización de esa fuente sísmica.

\section{Contexto tectónico}

La placa de Cocos se subduce perpendicular a la trinchera mesoamericana (TMA) originando un régimen de contracción en la placa superior (Mescua et al., 2017). Actualmente las variaciones morfotectónicas a lo largo del antearco de Costa Rica han sido atribuidas principalmente a cambios composicionales y morfológicos presentes en la placa de Cocos. Estos cambios han llevado a muchos autores a proponer una segmentación de la placa de Cocos, la cual ha sido definida por anomalías magnéticas (Barckhausen et al., 2001), batimetría multibeam (von Huene et al.,1995; von Huene et al., 2000), estudios sísmicos (Walther y Flueh 2002; Sallares y Charvis 2003; Walther, 2003; Wilson et al., 2003; Sallares et al., 2005) y muestreo geológico (Hauff et al., 1997; Werner et al., 1999; Wilson et al., 2003; Hoernle y Hauff, 2007).

Como resultado, en esta área se han definido tres segmentos de litosfera oceánica con edades variables, denominados GNS-1, GNS-2 y CNS-3, originados en el centro de expansión Cocos-Nazca, que limitan al norte con la Dorsal del Pacífico Este y al sur con la Placa Nazca.

El segmento CNS-1, que se subduce bajo el centro y sur de la Península de Nicoya, se caracteriza por una batimetría rugosa asociada a un fragmento de litosfera creada durante el Mioceno inferior (21-22,5 ma) (Barckhausen et al., 2001). El segmento CNS-2 comprende el dominio de los montes submarinos que chocan con la costa del centro y sur de Costa Rica e incluye la litosfera engrosada y rugosa del punto caliente de las Galápagos que contiene la Dorsal de Cocos (Gardner et al., 1992; Fisher et al., 1998). Este segmento exhibe una batimetría rugosa suave con numerosos montes submarinos cónicos y otras 
características batimétricas tales como el Plateau de Quepos en el centro (von Huene et al., 1995) y un área de corteza oceánica engrosada con un fondo marino elevado y un espesor de sedimentos variable de hasta $1 \mathrm{~km}$, asociado a la cresta de Cocos en el sector sur (Walther et al., 2000; von Huene et al., 2000; Kolarsky et al., 1995).

En cuanto a la sismicidad asociada al segmento CNS-2, Protti et al. (1995a) correlacionan el acoplamiento sísmico con las características del fondo marino subducido y observan que históricamente los montes submarinos aislados pueden reducir el acoplamiento entre las placas y generar sismos con una magnitud máxima de Mw 7.0. Superficialmente, la sismicidad es frecuente y de menor magnitud, lo cual sugiere que los altos batimétricos que ingresan a lo largo del margen central de Costa Rica forman asperezas aisladas que solo pueden generar sismos de tamaño moderado (Mw 6.0-7.3) (Protti et al., 1995b; Bilek et al., 2003; Proyecto Global Centroid Moment Tensor (GCMT), 2020).

Cada uno de estos montes submarinos subducidos deja una depresión a su paso, que puede ser rastreada hasta $55 \mathrm{~km}$ hacia el frente de deformación (Sak et al., 2009). Ese rastro es creado por la deformación y el levantamiento del talud continental cuando el monte submarino pasa por debajo de él, seguido por el colapso cuando se retira el soporte del área levantada (Hühnerbach et al., 2005). Las áreas de levantamiento sobre los montes submarinos se caracterizan por patrones complejos de fallas normales y de deslizamiento (Sak et al., 2009; Hühnerbach et al., 2005).

Estos montes submarinos pueden actuar como asperezas o barreras temporales en la zona de acople entre las placas (Watts et al., 2010), lo que dificulta la propagación de ruptura o genera esfuerzos locales que contribuyen a la ocurrencia de sismos fuertes en la zona. Husen et al., (2002) asocian el sismo del 25 de marzo de 1990, Mw 7.3 (GCMT, 2020) dentro del segmento CNS-2 en la entrada del Golfo de Nicoya, ubicado a $\sim 25 \mathrm{~km}$ al oeste del sismo del 24 de agosto de 2020, con un monte submarino que podría estar actuando como aspereza.
Entre los sismos más importantes ocurridos recientemente en la zona, se encuentran el del 13 de noviembre de 2017 a las 02:28:20.3 hora UTC, Mw 6.4, que se ubicó a $14 \mathrm{~km}$ al sureste del epicentro del sismo de Herradura o el sismo de Cóbano en marzo de 1990, -13:22:55 hora UTC, Mw 7.3, que también se ubicó cercano a este.

\section{Metodología}

Se realizó una caracterización de los parámetros de la fuente sísmica, para ello se utilizó principalmente el catálogo sísmico y formas de onda de la red sísmica del OVSICORI-UNA. Además, se revisaron los catálogos disponibles en línea del International Seismological Center (ISC) (2020), del Proyecto Global Centroide Momento Tensor (GCMT) (2020), del USGS (2020) y Guendel, (1986). Los sismos se localizaron con el programa GenLoc de Fan et al., (2006); para la inversión, el centroide y tensor de momento (CTM), se utilizó el paquete ISOLA (Sokos y Zahradnik, 2008 y 2013) y FOCMEG (Snoke et al., 1984), esto permitió obtener el mecanismo focal por medio de las polaridades de los primeros arribos de la onda $\mathrm{P}$. Para la distribución del deslizamiento en el plano de falla se implementó el paquete computacional de inversión lineal de desplazamiento (Gallovic y Zahradnik 2011; Gallovic et al., 2014). Para la localización de los sismos, cálculo del CMT, distribución de deslizamiento en la falla y mecanismo focal se usó el modelo de velocidad de Quintero y Kissling (2001) y en el caso del cálculo de cambio de esfuerzos estático de Coulomb ( $\triangle \mathrm{CFS}$ ) se empleó el paquete computacional Coulomb 3.3 (Toda et al., 2005; Lin et al., 2004). Se usó el método de cambio de esfuerzos con el fin de encontrar áreas donde se ve un aumento de $\triangle \mathrm{CFS}$ y señalar la posible ocurrencia de futuros eventos sísmicos.

Para el catálogo sísmico del OVSICORI-UNA, los sismos entre 1986-1992, fueron localizados con el programa HYPOINVERSE (Klein, 1984). A partir de 1992 hasta 03 de marzo del 2010 se 
Tabla 1. Intensidades instrumentales en la escala de Mercalli Modificada (MMI).

\begin{tabular}{|c|c|c|c|c|}
\hline $\begin{array}{c}\text { Intensidad } \\
\text { instrumental (IMM) }\end{array}$ & $\begin{array}{c}\text { Aceleración } \\
(\mathbf{\%} \mathbf{g})\end{array}$ & $\begin{array}{c}\text { Velocidad } \\
(\mathbf{c m} / \mathbf{s})\end{array}$ & $\begin{array}{c}\text { Sacudida } \\
\text { percibida }\end{array}$ & $\begin{array}{c}\text { Potencial de } \\
\text { daño }\end{array}$ \\
\hline I & $<0.17$ & $<0.1$ & No sentida & Ninguno \\
\hline II-III & $0.17-1.4$ & $0.1-1.1$ & Débil & Ninguno \\
\hline IV & $1.4-3.9$ & $1.1-3.4$ & Ligera & Ninguno \\
\hline V & $3.9-9.2$ & $3.4-8.1$ & Moderada & Muy ligero \\
\hline VI & $9.2-18$ & $8.1-16$ & Fuerte & Ligero \\
\hline VII & $18-34$ & $16-31$ & Muy fuerte & Moderado \\
\hline VIII & $34-65$ & $31-60$ & Severa & Moderado a fuerte \\
\hline XI & $65-124$ & $60-116$ & Violenta & Fuerte \\
\hline X & $>124$ & $>116$ & Extrema & Muy fuerte \\
\hline
\end{tabular}

procesó por medio del programa HYPOCENTER (Lienert y Haskov, 1995). Siendo el 04 de marzo del 2010, cuando las asociaciones de sismos y localizaciones fueron hechas con ANTELOPE (BRTT, 2010), el cual hace uso del programa de localización llamado LOCSAT (Bratt y Bache, 1988), basado en el modelo de velocidad IASPEI91 (Kennett y Engdahl, 1991) para sismos regionales y telesismos, y las librerías GENLOC (Generalized Earhquake-Location) de Fan et al. (2006), basado en el modelo de velocidad de Quintero y Kissling (2001) para la sismicidad local. El uso de un modelo $1 \mathrm{D}$ para la loacalización de sismos y la metodología de inversión puede producir que sismos superficiales aparezcan en el aire o en la capa de agua; estos sismos son un poco más profundos; pero en este trabajo son graficados en las localizaciones obtenidas sin hacerles modificación.

En el catálogo OVSICORI-UNA, las magnitudes de los sismos que ocurrieron antes del 10 de marzo del 2010 se obtuvieron de la siguiente forma: la magnitud de los eventos sísmicos fue obtenida del promedio de las magnitudes parciales calculadas para cada estación, según la duración de las trazas en las estaciones de corto período. Estas magnitudes parciales se calcularon según la relación obtenida por el Servicio Geológico de los Estados Unidos (USGS) para sismos en Alaska:

$M c=-1.16+2.01 * \log 10 \mathrm{~T}+0.0035 * \Delta+\Delta \mathrm{M}$

T: duración del sismo

$\Delta$ : distancia epicentral

$\Delta M$ : corrección para cada estación
A partir de 1992 se implementó un sistema digital en el centro de registro, que además contaba con estaciones de banda ancha, con respuestas instrumentales calibradas.

Estas permitían simular la respuesta de un instrumento Wood-Anderson para el cálculo de la magnitud local Richter. En este cálculo se utiliza la siguiente relación propuesta por Hutton y Boore (1987):

$M L=\log 10(\mathrm{~A})+1.11 * \log 10(\Delta)+0.00189 * \Delta$ $-2.09$

$\Delta$ : Distancia epicentral.

A: Amplitud pico-pico de la onda.

Después de implementado el sistema de adquisición sismológico ANTELOPE, se dejó de cuantificar la magnitud coda y, en vez de esta y para sismos locales, se cuantifica la magnitud local (Ml) que representa la magnitud Richter, donde el paquete computacional (BRTT, 2010) aplica la metodología y funciones de corrección propuestas por Richter (1935). Es a partir de la implementación de ANTELOPE que se comienza con el cálculo de la magnitud momento de la onda P: Mwp (Tsuboi et al., 1995). Las magnitudes son calculadas automáticamente por el paquete ANTELOPE y se reporta la mediana de los valores de magnitud cuantificados en cada estación. En los años anteriores, se calculaba el valor medio de las magnitudes obtenidas en cada estación. Las magnitudes calculadas según sea la metodología van a variar; para el caso del sismo 
del 11 de noviembre del 2017, USGS (2020) y GCMT (2020) lo reportan con magnitudes entre 6.1 y 6.6 y el OVSICORI-UNA con Ml 6.4 y en este estudio se encontró $\mathrm{Mw}$ 6.4; y la diferencia con otros estudios que hacen calculo de magnitud momento global es por el ancho de banda usado en la inversión; siendo usadas frecuencias más altas en el caso de usar datos locales. Para el sismo del 24 de agosto de 2020, el GCMT (2020) y USGS (2020) reportaron magnitudes entre 5.5 y 6.1 y el OVSICORI-UNA con Ml 6.1, Mwp 6.3 y en este trabajo se encuentra magnitud momento usando datos locales de $\mathrm{Mw}$ 5.9.

El mapa de intensidades instrumentales, para el sismo de Herradura, en la escala de Mercalli Modificada (MMI) se generó mediante una interpolación estadística (Krigging) a través del software Arc Gis 10.7, para ello se utilizaron las aceleraciones pico (PGA) registradas en 21 estaciones distribuidas en el territorio nacional.
La conversión de PGA a MMI se llevó a cabo aplicando la conversión propuesta por Wald et al. (2005) (Tabla 1).

Los resultados obtenidos fueron comparados con los reportes de la población en la página oficial del Facebook del OVSICORI-UNA para determinar la relación entre las intensidades instrumentales en la escala MMI y las características de cómo fue sentido el sismo en diferentes ciudades y poblados del país.

\section{Resultados}

\subsection{RÉPLICAS DEL SISMO DE HERRADURA}

El sismo de Herradura de las 21:51:08.1, hora UTG, se localizó a una latitud 9.501 y longitud -84.665, a una profundidad de $18 \mathrm{~km}$, con magnitud Ml 6.1 . Las réplicas de este evento (para dos

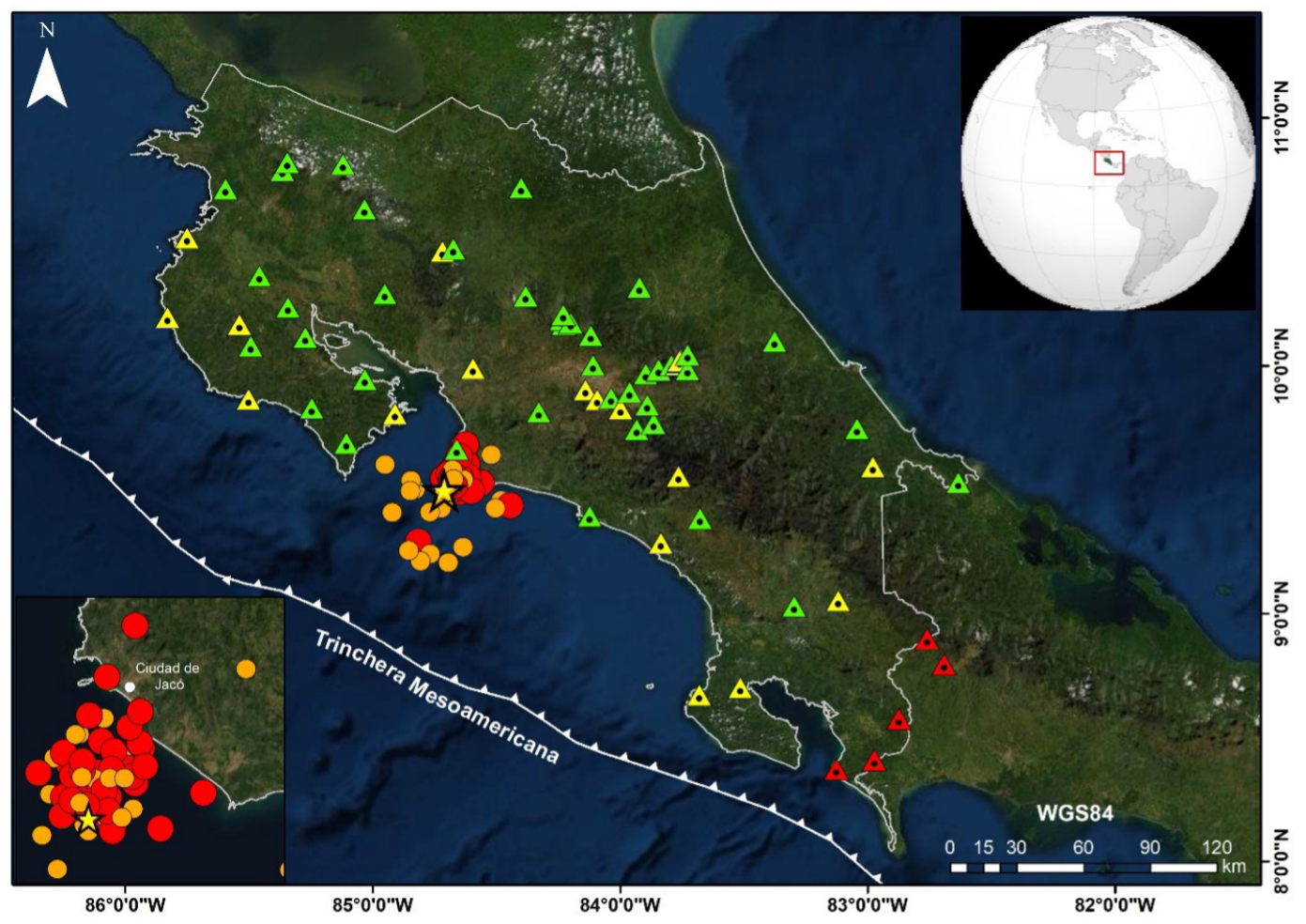

Figura 1 Sismicidad asociada al sismo de Herradura, el sismo principal se indica por una estrella amarilla y las réplicas por círculos, donde el color indica la profundidad, los anaranjados con profundidades entre 0 y $20 \mathrm{~km}$ y los rojos presentan profundidades mayores a $20 \mathrm{~km}$. Las estaciones sísmicas usadas en la localización del sismo principal se indican por triángulos, el color indica el residuo; verde si es menor a 0.5 segundos, amarillo entre 0.5 y 1.5 segundos y rojo para residuos mayores a 1.5 segundos. La estación sísmica más cercana al epicentro del sismo principal está ubicada en Herradura, Jacó. Para el cálculo de las réplicas también se usaron estas estaciones sísmicas. 
días posteriores) fueron localizadas principalmente frente a la costa del Pacífico Central de Costa Rica, en un área de $400 \mathrm{~km}^{2}$ aproximadamente (Figura 1). En total se registraron 91 réplicas cuyas magnitudes se encontraron entre Ml -0.8 y Ml 3.6. Referente a las profundidades, estas oscilaron entre 3.5 y $32.7 \mathrm{~km}$ del total de esas réplicas, 80 se ubicaron a profundidades mayores a $15 \mathrm{~km}$, concentradas en una banda de 20 a $25 \mathrm{~km}$ de profundidad y con epicentros desde la costa hacia la TMA.

\subsubsection{MECANISMO FOCAL DEL SISMO DE HERRADURA}

La solución del mecanismo focal (MF) del evento principal, calculado usando polaridades de los primeros arribos y el programa FOCMEC (Snoke et al., 1984) muestran un plano de falla inclinado al NE con un ángulo de $31^{\circ}$, el cual corresponde a una falla inversa con azimut de $287^{\circ}$ y ángulo de deslizamiento de $71^{\circ}\left(287^{\circ} / 31^{\circ} / 71^{\circ}\right)$.

La solución del MF obtenida de la inversión de formas de onda y el paquete ISOLA (Sokos y Zahradnik, 2008 y 2013), también da como resultado un mecanismo inverso, con un plano de falla buzando al NE, con ángulo de inclinación de $20^{\circ}$, rumbo de $311^{\circ}$ y deslizamiento de $98^{\circ}$ $\left(311^{\circ} / 20^{\circ} / 98^{\circ}\right)$ (Figura 2B). El sismo está relacionado con el acople entre la placa de Cocos y la placa Caribe (Co-Ca) en el Pacífico Central de Costa Rica.

La correlación entre los datos sintéticos y observados usando estaciones en el campo cercano entre 18 y $129 \mathrm{~km}$ del epicentro es alta (Figura 2A) y el mecanismo focal obtenido está en concordancia con los mecanismos calculados por el USGS
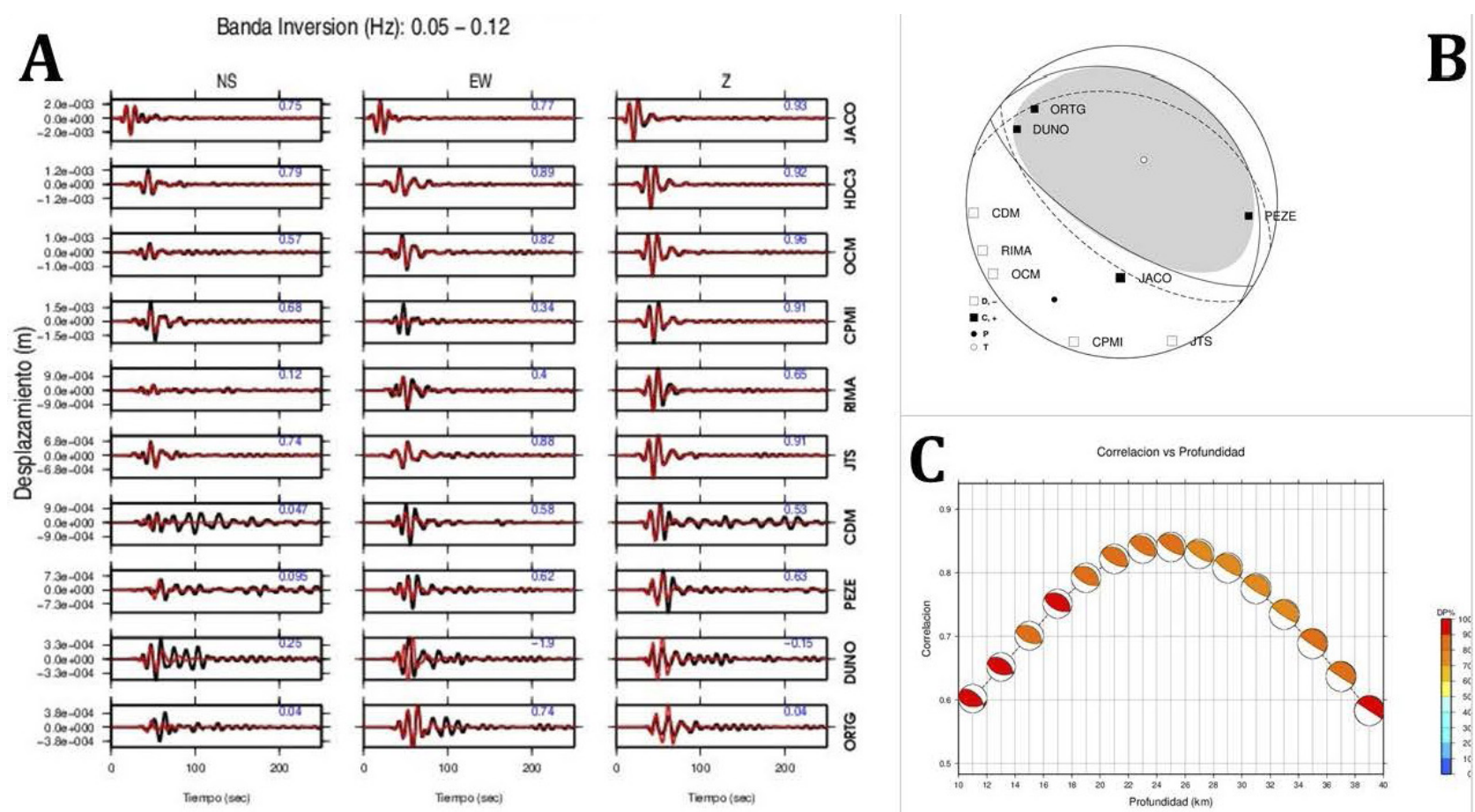

Figura 2 A) Correlación de las formas de onda de desplazamiento reales (negro) y sintéticas (rojo) usando un ancho de banda de 0.05 a $0.12 \mathrm{~Hz}$. El gráfico para cada componente de la estación sísmica indica tiempo (segundos) vs desplazamiento (metros), B) Mecanismo focal calculado usando inversión de formas de onda (línea continua en la esfera focal) y polaridades de primeros arribos, indicando los planos de falla con líneas discontinuas. Las estaciones usadas en la inversión de las formas de onda muestran la respectiva polaridad; con rectángulos blancos se indica la dilatación (D) y con rectángulos negros la compresión (C), el eje de Presión (P) está indicado por un círculo negro y el de tensión (T) por un círculo blanco. El eje de presión $\left(215^{\circ} / 25\right)$ tiene dirección noreste y el de tensión con parámetros $27^{\circ} / 65^{\circ}$, C) Correlación vs profundidad para el sismo de Herradura. El porcentaje doble par de fuerzas (DP) se indica por una barra de color a la derecha y en este caso es de $81 \%$ DP. 
Tabla 2. PGA en estaciones del OVSICORI-UNA.

\begin{tabular}{|c|c|c|c|c|}
\hline Estación & E (\%g) & $\mathrm{N}(\% \mathrm{~g})$ & $\mathrm{Z}(\% \mathrm{~g})$ & Intensidad \\
\hline & Valor máximo & Valor máximo & Valor máximo & I-XII \\
\hline BATAN & 0.74 & 0.63 & 0.18 & III \\
\hline CCOL & 0.80 & 0.56 & 0.04 & III \\
\hline CDITO & 0.18 & 0.24 & 0.14 & III \\
\hline COVE & 0.47 & 0.56 & 0.21 & III \\
\hline $\mathrm{CDM}$ & 1.03 & 0.99 & 0.57 & III \\
\hline CPMI & 0.78 & 0.55 & 0.33 & III \\
\hline CTCR & 0.59 & 0.62 & 0.22 & III \\
\hline DUNO & 1.19 & 1.78 & 0.50 & IV \\
\hline HDC3 & 0.34 & 0.32 & 0.29 & III \\
\hline JACO & 11.52 & 11.03 & 3.76 & VI \\
\hline JTS & 1.09 & 0.65 & 0.30 & III \\
\hline $\mathrm{OCM}$ & 0.85 & 0.75 & 0.31 & III \\
\hline ORTG & 0.11 & 0.14 & 0.11 & II \\
\hline PEZE & 0.93 & 1.37 & 0.31 & III \\
\hline PNPB & 0.39 & 0.33 & 0.13 & III \\
\hline POTG & 0.58 & 0.60 & 0.16 & III \\
\hline RIFO & 0.53 & 0.40 & 0.21 & III \\
\hline RIMA & 1.29 & 0.99 & 0.35 & III \\
\hline RIOS & 1.97 & 2.10 & 0.77 & IV \\
\hline TIGR & 0.86 & 0.70 & 0.32 & III \\
\hline VMAR & 0.33 & 0.01 & 0.25 & III \\
\hline
\end{tabular}

(2020). Asimismo, el centroide calculado usando datos de la red del OVSICORI-UNA, se ubicó debajo del epicentro a una profundidad de $25 \mathrm{~km}$ y +1.3 segundos después del tiempo de origen y una magnitud Mw 5.9.

Además, la correlación por profundidad también es bastante alta, alcanzando un valor de 0.83 (Figura 2C) para $25 \mathrm{~km}$ de profundidad; la cual corresponde a una profundidad similar a los sismos ubicados entre la interfaz CO-CA para esta zona.

Se hizo búsqueda en el plano, pero la mejor correlación se obtuvo para un punto ubicado por debajo del epicentro; lo que indica que los deslizamientos en la falla fueron focalizados y no se produjo rompimiento a lo largo del plano de falla, como lo evidencian las réplicas, que se mantuvieron concentradas y cercanas al hipocentro.

\subsubsection{INTENSIDADES INSTRUMENTALES EN LA ESCALA DE MERCALLI MODIFICADA (MMI)}

Las máximas PGA se registraron en la estación sísmica JACO, ubicada en Herradura, Garabito, Puntarenas, estas alcanzaron $11.52 \% \mathrm{~g}$, que corresponde a una intensidad de VI en la escala IMM. Sin embargo, sismos con estas características tienen la particularidad de no producir daños importantes en infraestructuras, aunque la sacudida es fuerte y sentida por la población. 
Para establecer la relación planteada por Wald et al., (2005) se utilizaron datos de PGA de 21 estaciones sísmicas, distribuidas por todo el país, y se determinó que las intensidades se corresponden con valores entre II y VI (Tabla 2, Figura 3B).

Las mayores intensidades (VI) se concentraron en la ciudad de Jacó y en Herradura; en Cóbano y Paquera, en el extremo sureste de la Península de Nicoya, se registró una intensidad máxima de $\mathrm{V}$, las intensidades menores a III se registraron en poblados como San Isidro del General, Limón, Upala y en ciudades de la Gran Área metropolitana (GAM) destacando centros urbanos como San José (capital del país), Alajuela, Cartago y Heredia (Figura 3A).

Con base en la información recabada en el Facebook del OVSICORI-UNA se identificaron algunas particularidades relevantes, ya que la población reportó el sismo sentido desde muy fuerte a fuerte. Se destaca que en la ciudad de Jacó, ubicada a $13 \mathrm{~km}$ del epicentro, se reportó la caída de objetos en supermercados. Con la información facilitada por los usuarios de la red social (Facebook oficial del OVSICORI-UNA) se lograron sistematizar las principales características del sismo en las siete provincias del país (Tabla 3). Se hace énfasis en que la información recabada correspondió a reportes en el Facebook del
OVSICORI-UNA y no a encuestas. En total se registraron 2679 reportes, los cuales se clasificaron por provincia y según la descripción macrosísmica.

\section{Análisis de la sismicidad en el área de estudio}

En el área de estudio se han registrado otros sismos importantes, tales como a) el sismo del 25 de marzo de 1990 a las 13:22:55 hora UTC, Mw 7.3 (GCMT, 2020), b) el sismo del 20 de agosto 1999 a las 10:02:20 hora UTC, Mw 6.9 (GCMT, 2020) y c) el sismo del 13 de noviembre 2017, Mw 6.4 (este trabajo) y se presentó a las 02:28:20 hora UTC (Figura 4). Algunos de los sismos registrados en el área presentan dos particularidades importantes: a) microsismicidad abundante y b) que en un período de tiempo corto los eventos premonitores y sus réplicas, alcanzan una magnitud significativa, incluso en ocasiones cercanas a la del evento principal. Por ejemplo, el sismo ocurrido el 21 diciembre de 1939 (20:54:53, hora UTC) con magnitud Ms 7.3, y al día siguiente (04:44:03 hora UTC) se presentó una réplica magnitud Ms 6.8; el 19 de julio 1956 (23:26:30 y 23:38:04 hora UTC) se presentaron dos sismos de igual magnitud (Ms 6.2). El 23 agosto de 1978 (00:38:31 y 00:50:27
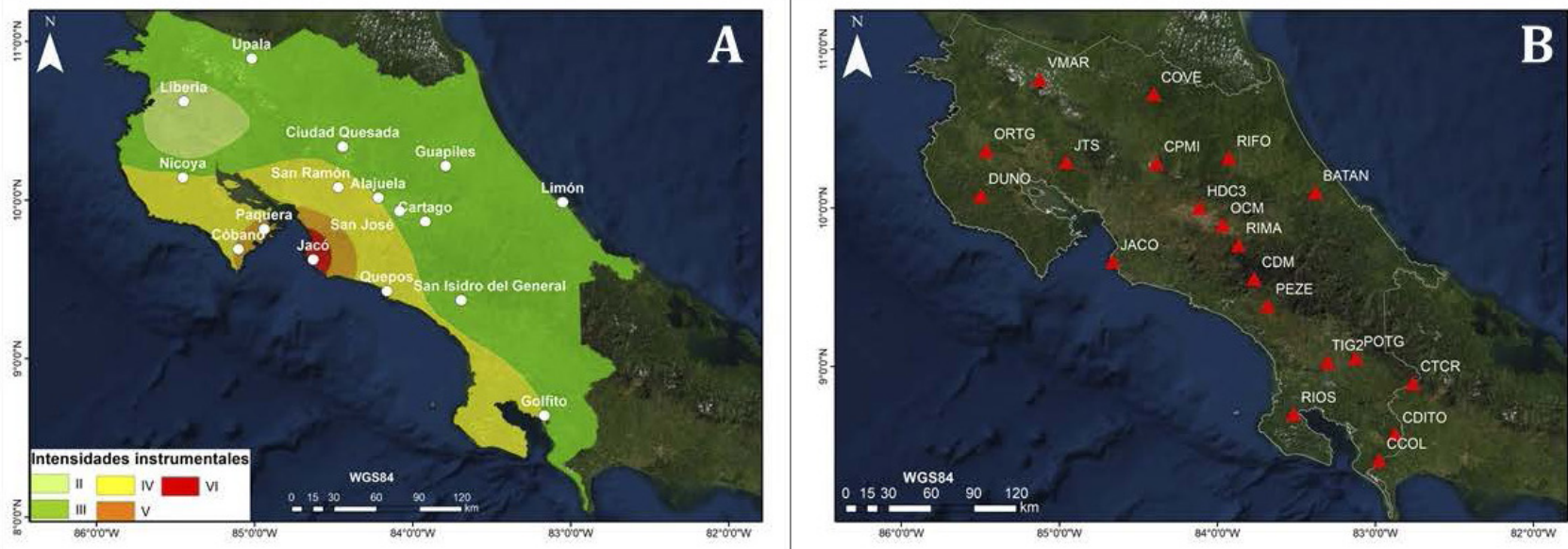

Figura 3 A) Intensidades instrumentales en la escala de Mercalli Modificada (MMI) registradas para el sismo de Herradura del 24 de agosto de 2020, MI 6,1. Para determinar las intensidades IMM se utilizó la relación de Wald et al., 2005; para conversión de PGA a intensidad. Los puntos blancos indican ciudades y poblados, B) Estaciones sísmicas, señaladas con triángulos rojos, donde se registraron las intensidades instrumentales. 
Tabla 3. Descripción macrosísmica del sismo de Herradura y su distribución por provincias.

\section{Provincia}

\begin{tabular}{|c|c|}
\hline Provincia & Ciudades o poblados \\
\hline \multirow{4}{*}{ Alajuela } & Ciudad de Alajuela \\
\hline & Turrucares \\
\hline & $\begin{array}{l}\text { Poás, Naranjo, La Garita, Ciudad Quesada, } \\
\text { Sarchí, Río Segundo de Alajuela, Grecia, } \\
\text { San Ramón, Palmares y La Fortuna de San } \\
\text { Carlos. }\end{array}$ \\
\hline & Guácima de Alajuela \\
\hline \multirow[t]{2}{*}{ Cartago } & $\begin{array}{l}\text { Ciudad de Cartago, Orosí, Caballo Blanco, } \\
\text { Tres Ríos de La Unión y Villas de Ayarco. }\end{array}$ \\
\hline & Juan Viñas \\
\hline \multirow[t]{3}{*}{ Heredia } & $\begin{array}{l}\text { Ciudad de Heredia, Santo Domingo, San } \\
\text { Rafael, San Pedro de Barva y San Pablo. }\end{array}$ \\
\hline & Varablanca \\
\hline & Mercedes Norte y Barva \\
\hline \multirow[t]{2}{*}{ Guanacaste } & Liberia, Sardinal, Nosara \\
\hline & $\begin{array}{l}\text { Tilarán, Nandayure, Cañas, Guayabo y Santa } \\
\text { Cruz. }\end{array}$ \\
\hline \multirow[t]{3}{*}{ Limón } & $\begin{array}{l}\text { Ciudad de Limón, Siquirres, Cocles y Pueblo } \\
\text { Nuevo de Guácimo. }\end{array}$ \\
\hline & Río Jiménez de Guácimo \\
\hline & Guápiles y Pensurth \\
\hline \multirow[t]{7}{*}{ Puntarenas } & Ciudad de Puntarenas, Barranca \\
\hline & Monteverde \\
\hline & Parrita \\
\hline & San Vito \\
\hline & Quepos y Esparza \\
\hline & Río Claro de Golfito, \\
\hline & Orotina, ciudad de Jacó, Herradura y Uvita \\
\hline \multirow[t]{2}{*}{ San José } & $\begin{array}{l}\text { Ciudad de San José, Pavas, Moravia } \\
\text { Guadalupe, Santa Ana, San Antonio de } \\
\text { Coronado, Alajuelita, Tibás, San Pablo León } \\
\text { Cortés, San Francisco de Dos Ríos, Sabanilla } \\
\text { Montes de Oca, Desamparados, Pérez } \\
\text { Zeledón y Acosta. }\end{array}$ \\
\hline & Vueltas del Jorco, Aserrí \\
\hline
\end{tabular}

\section{Descripción macrosísmica}

Sumamente fuerte y largo. Movimiento de objetos y caída de ellos en algunas viviendas.

Demasiado fuerte y largo. La población reportó que percibieron dos sismos largos de abajo hacia arriba y luego hacia los lados.

Muy fuerte y de larga duración.

Se sintió hacia arriba y abajo y después hacia los lados. Larga duración.

Muy fuerte y largo, en la ciudad de Cartago se reportó movimiento de objetos.

Muy fuerte y duró aproximadamente 13 segundos.

Muy fuerte y de larga duración, en San Rafael se reportó movimiento de objetos.

Muy leve pero de larga duración.

Se reportó como dos sismos sentidos muy fuertes y de larga duración.

Leve pero de larga duración.

Fuerte y de larga duración.

Fuerte y de larga duración.

Muy fuerte.

Leve, en Guápiles provocó el movimiento de objetos.

Muy fuerte

Fuerte y de larga duración, movimiento de objetos.

Muy fuerte y de larga duración, se reportaron objetos caídos y quebrados.

\section{Leve.}

Fuerte y de larga duración.

Leve y de larga duración.

Muy fuerte y de larga duración.

Fuerte y de larga duración. En Moravia reportaron sentir dos sismos, una más fuerte que el otro. En Sabanilla Montes de Oca se reportó la caída de objetos.

Muy fuerte y de larga duración. 
hora UTG), se ubicaron dos eventos en Sámara, frente a la Península de Nicoya, con magnitud de Ms 7.0 y 6.9, respectivamente; el 25 de marzo de 1990 (13:16:05 hora UTC), ocurrió un evento premonitor de Ms 6.4 y un evento principal de $\mathrm{Mw}$ 7.3 a las 13:22:55 hora UTC; el 20 de agosto de 1999 (10:02:20 hora UTC), un sismo de magnitud Mw 6.9 y una réplica al día siguiente (10:49:50 hora UTC) de Mw 5.8.

El penúltimo evento fuerte registrado en esta área corresponde al sismo de Esterillos del 13 de noviembre de 2017 (02:28:20 hora UTC), con magnitud Mw 6.4 y con una réplica de Ml 5.2 a las 02:32:31 hora UTG (Güendel, 1986; ISG, 2020; OVSICORI-UNA, 2020; Global Catalogue, 2020; para información sobre sismos históricos en la zona) (Tabla 4).

Referente al sismo de Esterillos, se obtuvo que los parámetros de la fuente sísmica lo ubican en la latitud 9.463 y longitud -84.546, con una profundidad de $27 \mathrm{~km}$. La magnitud es de Ml 6.4, con el centroide a $21 \mathrm{~km}$ de profundidad debajo del epicentro y a +4.0 segundos después del tiempo de origen. El plano de falla se orienta en dirección $289^{\circ}$, y buza $17^{\circ}$, su deslizamiento es de $85^{\circ}$ (Figura 5). La magnitud momento obtenida usando datos de la red local es dos décimas inferiores a la obtenida usando datos de la red global por el GCMT (2020), que la ubica en Mw 6.6, cuya diferencia en magnitud se debe al ancho de frecuencias usadas en la inversión. Por otra parte, se muestran (Figura 6A) los epicentros de algunos sismos registrados entre el 2010 y agosto de 2020, con su respectivo perfil indicado a lo largo de la línea 1, así como la sismicidad asociada a los sismos de Esterillos y Herradura. La sismicidad se concentró en los primeros $25 \mathrm{~km}$ de profundidad (Figura 6B), con epicentros al sur de la ciudad de Jacó. Un perfil de la sismicidad a lo largo de la línea 1 muestra que el sismo se encuentra en la parte superior de la

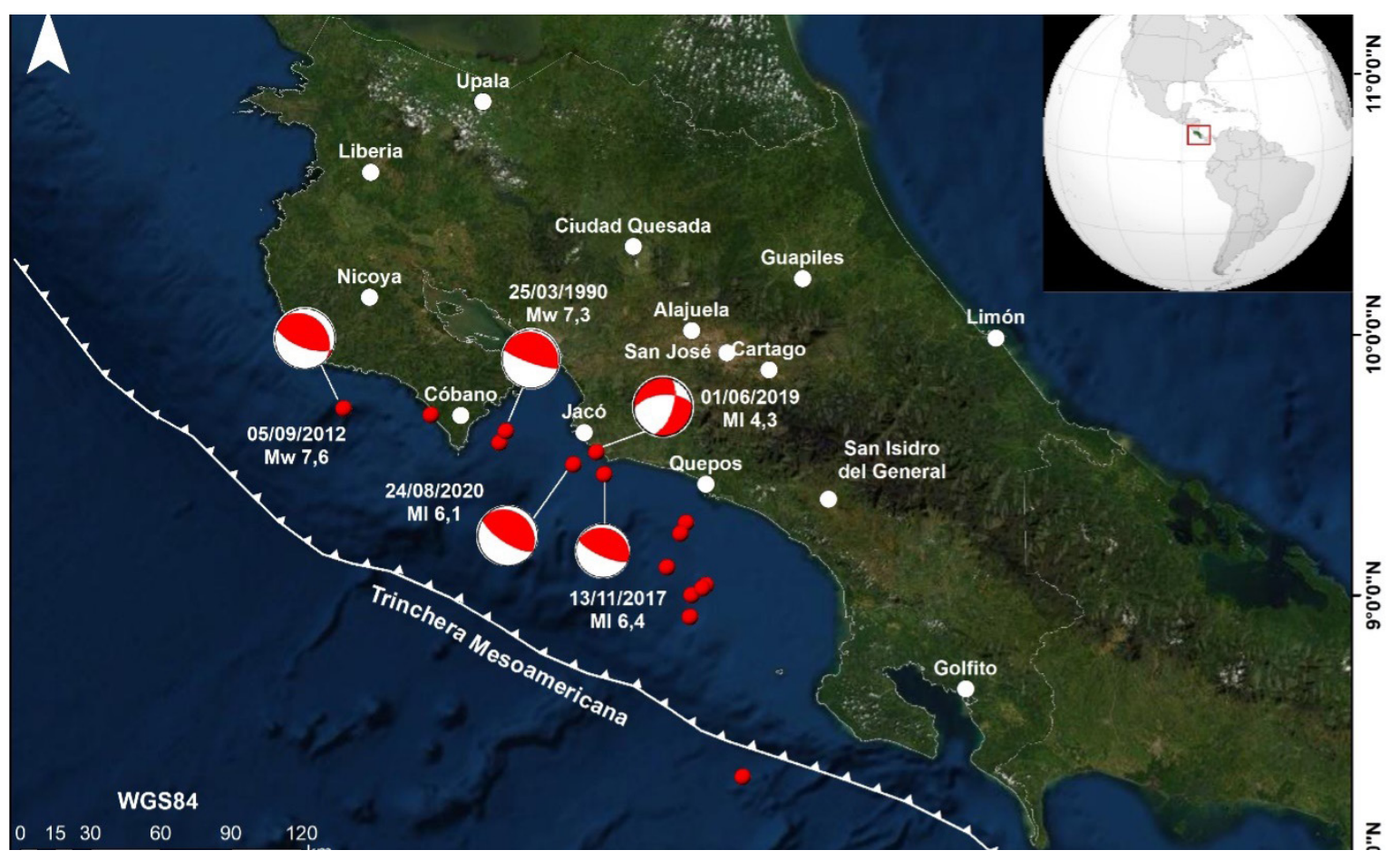

Figura 4 Se muestran con círculos rojos los epicentros para sismos que se han registrado en el Pacífico Central de Costa Rica, desde 1984 hasta agosto de 2020, con magnitudes $\mathrm{MI}>5.9$. Se destacan cuatro principales eventos con su respectiva fecha y magnitud asociada a) el sismo del 25 de marzo de 1990 (Mw 7.3), b) el sismo del 05 de septiembre de 2012 (Mw 7.6), conocido como el terremoto de Nicoya, c) el sismo del 13 de noviembre de 2017 (MI 6.4) y d) el sismo del 01 de junio de 2019 (MI 4.3 ), e) el sismo del 25 de agosto de 2020 (MI 6,1). Los MF para el sismo del 05 setiembre 2012 (Quintero et al., 2014) y para el sismo de 25 de marzo 1990 (GCMT, 2020 ); los otros tres se realizaron en este trabajo. Los MF indican que los sismos ocurren como consecuencia de fallas inversas asociadas a la subducción de la placa de Cocos bajo la placa Caribe, buzando al nor-noreste y en bajo ángulo. 
Tabla 4. Sismos históricos importantes entre 1939 y 2020.

\begin{tabular}{|c|c|c|c|c|c|}
\hline Sismo & Año & $\begin{array}{c}\text { Mes- } \\
\text { día }\end{array}$ & Hora UTC & Latitud & Longitud \\
\hline $\mathbf{1}$ & 1939 & $12-21$ & $20: 54: 47.20$ & 10.140 & -84.630 \\
\hline $\mathbf{2}$ & 1939 & $12-22$ & $04: 43: 58.10$ & 09.800 & -84.550 \\
\hline $\mathbf{3}$ & 1956 & $07-19$ & $23: 26: 37.00$ & 09.550 & -84.140 \\
\hline $\mathbf{4}$ & 1956 & $07-19$ & $23: 38: 14.00$ & 09.520 & -83.960 \\
\hline $\mathbf{5}$ & 1978 & $08-23$ & $00: 38: 27.00$ & 09.760 & -85.570 \\
\hline $\mathbf{6}$ & 1978 & $08-23$ & $00: 50: 24.40$ & 09.890 & -85.500 \\
\hline $\mathbf{7}$ & 1990 & $03-25$ & $13: 16: 05.30$ & 09.585 & -84.948 \\
\hline $\mathbf{8}$ & 1990 & $03-25$ & $13: 22: 55.20$ & 09.628 & -84.923 \\
\hline $\mathbf{9}$ & 1999 & $08-10$ & $14: 55: 42.80$ & 09.263 & -84.120 \\
\hline $\mathbf{1 0}$ & 1999 & $08-10$ & $14: 55: 42.80$ & 09.263 & -84.120 \\
\hline $\mathbf{1 1}$ & 1999 & $08-20$ & $10: 02: 20.00$ & 09.038 & -84.156 \\
\hline $\mathbf{1 2}$ & 1999 & $08-20$ & $10: 42: 15.60$ & 08.884 & -84.107 \\
\hline $\mathbf{1 3}$ & 1999 & $08-21$ & $10: 49: 50.00$ & 08.976 & -84.014 \\
\hline $\mathbf{1 4}$ & $\mathbf{2 0 1 7}$ & $\mathbf{1 1 - 1 3}$ & $\mathbf{0 2 : 2 8 : 2 0 . 3 0}$ & $\mathbf{0 9 . 4 6 3}$ & $\mathbf{- 8 4 . 5 4 6}$ \\
\hline $\mathbf{1 5}$ & 2017 & $11-13$ & $02: 32: 30.90$ & 09.454 & -84.546 \\
\hline $\mathbf{1 6}$ & 2019 & $06-01$ & $06: 53: 29.20$ & 09.542 & -84.573 \\
\hline $\mathbf{1 7}$ & $\mathbf{2 0 2 0}$ & $\mathbf{0 8}-\mathbf{2 4}$ & $\mathbf{2 1 : 5 1 : 0 8 . 0 0}$ & $\mathbf{0 9 . 5 0 1}$ & $\mathbf{- 8 4 . 6 6 5}$ \\
\hline $\begin{array}{l}\text { Guendel, (1986) } \\
\text { International Seismological Centre, (2020) }\end{array}$ \\
\hline USGS, (2020) \\
OVSICORI-UNA, (2020) \\
Dziewonski et al., (1981), Ekström et al., (2012)
\end{tabular}

\begin{tabular}{|c|c|c|c|}
\hline Profundidad & Magnitud & Magnitud & Fuente \\
\hline 33.0 & 7.3 & 7.6 MISC & 1,2 \\
\hline 33.0 & 6.8 & 6.8 SISC & 1,2 \\
\hline 93.0 & 6.2 & 5.8 SISC & 1,2 \\
\hline 81.0 & 6.2 & & 1,2 \\
\hline 26.0 & 7.0 SUSGS & & 1,3 \\
\hline 42.0 & 6.9 SISC & & 1,2 \\
\hline 27.9 & 6.4 SUSGS & & 4,3 \\
\hline 23.3 & 7.3 WGCMT & & 4,5 \\
\hline 35.0 & 5.7 LUNA & 5.5 WGCMT & 4,5 \\
\hline 35.0 & 5.7 LUNA & 5.5 WGCMT & 4,5 \\
\hline 14.0 & 6.2 LUNA & 6.9 WGCMT & 4,5 \\
\hline 21.6 & 5.3 LUNA & $5.2 b U S G S$ & 4,3 \\
\hline 45.3 & 5.8 WGCMT & & 4,5 \\
\hline $\mathbf{2 7 . 0}$ & 6.4LUNA & & 4 \\
\hline 17.3 & 5.2 LUNA & & 4 \\
\hline 12.3 & 4.3 LUNA & & 4 \\
\hline 18.3 & 6.1LUNA & & 4 \\
\hline
\end{tabular}

zona sismogénica y en contacto CO-CA y el sismo principal corresponde a una falla inversa. El sismo de Esterillos se caracteriza por tener réplicas hacia arriba del epicentro y el de Herradura por estar alrededor de la zona epicentral.

Es posible que estos sismos estén asociados con la subducción de los altos batimétricos que ingresan a lo largo del margen central de Costa Rica y que forman asperezas aisladas que pueden generar terremotos que podrían alcanzar una magnitud Mw 7.3 (GGMT, 2020).

Después del sismo de Esterillos (Mw 6.4) se ubicó sismicidad cortical superficial, donde ocurrió un sismo magnitud Ml 4.3 el 01 de junio de 2019 a las 06:53:29 hora UTC, cuyo origen es atribuido a una falla normal con componente de desplazamiento y parámetros para un plano nodal azimut $=18^{\circ}$, buzamiento $=61^{\circ} \mathrm{y}$ deslizamiento $=$ $-139^{\circ}$, parámetros obtenidos con inversión por el CMT usando datos sísmicos de la red del OVSICORI-UNA. En el pasado sismicidad como la del sismo de Cóbano del 25 de marzo 1990, Mw 7.3 disparó sismicidad en la placa continental; que

ocurrió en superficie (Protti et al., 1995), después del sismo del 13 de noviembre de 2017 no es muy clara en una relación directa con este; pero existe sismicidad localizada en la placa continental, principalmente en fuentes sísmicas, cercanas al epicentro.

En profundidad y a unos $100 \mathrm{~km}$ de distancia de la trinchera, en la zona de contacto de las placas CO-CA se localizan pocos sismos y los sismos como el de Esterillos y el de Herradura ubican el centroide por debajo del epicentro, con réplicas hacia la TMA; por lo que la zona que evidencia poca sismicidad puede ser una barrera que impida rompimiento a lo largo del plano de inmersión de la falla (Figura 6). El cambio de esfuerzos estáticos de Coulomb para los sismos de Esterillos (Mw 6.4) y de Herradura (Mw 5.9) graficados con perfiles que cortan la TMA y en dirección al buzamiento de la placa de Cocos muestran que existe una acumulación de esfuerzos en los extremos superior e inferior de la falla inversa que originó estos sismos (Figura 7). El extremo inferior de esta zona de interfaz sismogénica entre las placas de Cocos y 
Caribe, ubicada al noroeste de donde ocurrieron los sismos en mención, ha mostrado poca sismicidad en el pasado y donde existe acumulación positiva de esfuerzos puede indicar una zona con potencial para generar futuros eventos sísmicos.

Resultados preliminares de la distribución de deslizamiento final en el plano de falla 289॰17\%/85 para el sismo de Esterillos, y para el sismo de Herradura en el plano de falla $311 \% / 20 \circ / 98^{\circ}$, muestran una concentración de deslizamientos concéntricos alrededor del centroide con deslizamientos máximos de 0.6 y 0.1 metros, respectivamente y con una ligera concentración hacia la parte inferior de la falla para el sismo de Esterillos y hacia la superficie para el sismo de Herradura (Figura 8).

\section{Discusión}

El sismo del 24 de agosto del 2020, 21:51:08 hora UTC, conocido como el sismo de Herradura, ubicado en el Pacífico Central de Costa Rica, corresponde a una falla inversa, con hipocentro a $18 \mathrm{~km}$ de profundidad, su centroide se encuentra debajo del epicentro a $25 \mathrm{~km}$ de profundidad y +1.3 segundos después del tiempo de origen. Sismos con este tipo de mecanismo focal están asociados a la interfaz Cocos-Nazca, tal y como se observa en el perfil en profundidad (Figura 6). La ubicación del centroide y de las réplicas muestran que los hipocentros se encuentran concentrados en un área pequeña y que no existe rompimiento a lo largo de la inmersión de la placa de Cocos bajo la placa Caribe.

Lo mismo ocurrió con el sismo de Esterillos con centroide por debajo del epicentro.

Estos sismos inversos de mayor magnitud están asociados con la subducción de los altos batimétricos que ingresan a lo largo del margen central del antearco de Costa Rica y forman asperezas aisladas que tienen potencial para generar sismos con una magnitud de Mw 7.3 (GCMT, 2020). El modelo de deformación de la placa superior
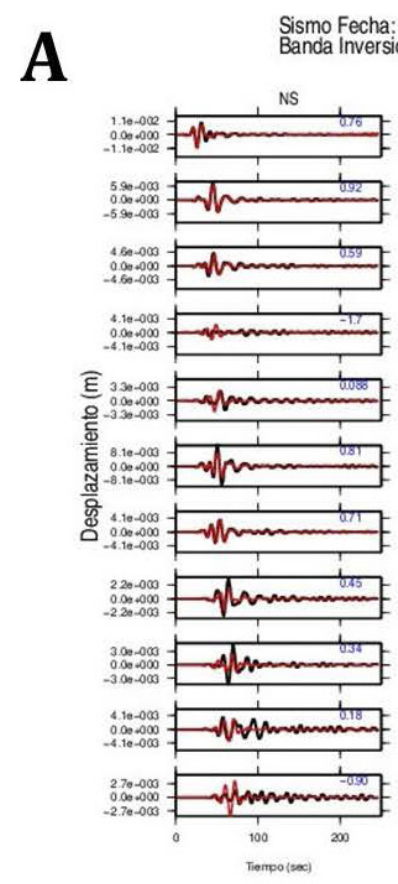

Sismo Fecha: 13/11/17 02:28:20.00
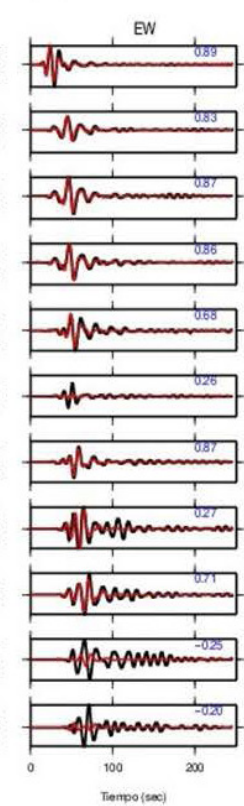

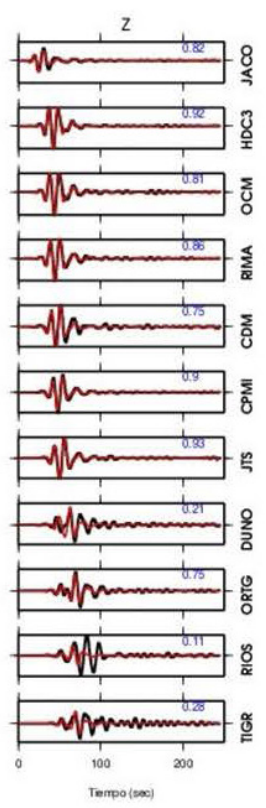

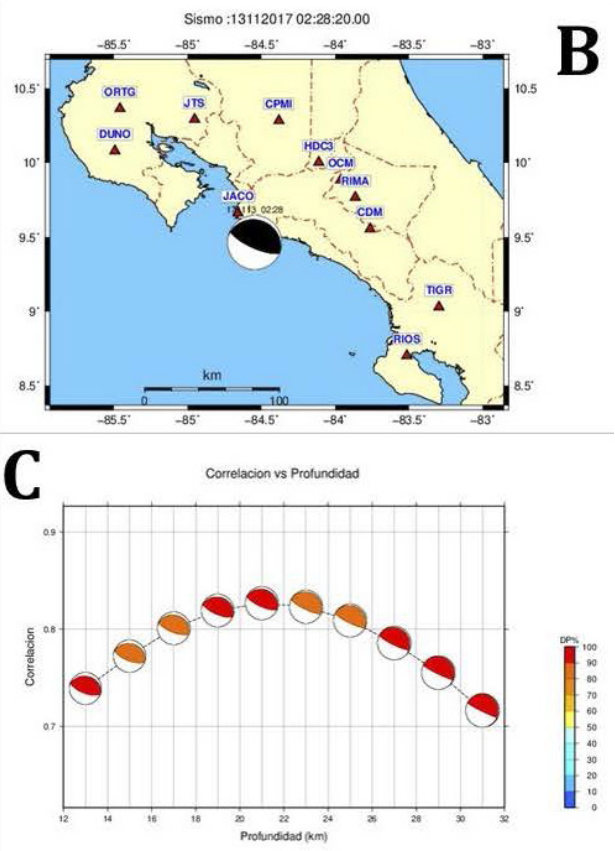

Figura 5 Inversión por el centroide y tensor de momento del sismo del 13 de noviembre de 2017. A) Gráfico de formas de ondas observadas y sintéticas en estación sísmicas usadas, en color negro y rojo, respectivamente, B) Mecanismo focal y estaciones usadas en la inversión, C) Gráfico que muestra la correlación a diferentes profundidades, con diagrama de barras indicando el porcentaje de doble par de fuerzas. 
propuesto para esta región por Domínguez et al. (1998) indica que se desarrollan fallas inversas arqueadas delante del monte submarino, fracturas subverticales que se extienden desde arriba del monte submarino, algunas con movimiento de desplazamiento de rumbo.

El monte submarino acrecionado proporciona una aspereza local, pero podría transferir la tensión a las fracturas preexistentes en la parte superior, en relación directa con los sismos que abarcan un amplio rango de profundidades.

Estos sismos con mecanismo focal normal estarían asociados al colapso producido por la depresión creada durante la deformación del talud continental cuando el monte submarino pasa por debajo, similar a los modelos propuestos por Dominguez et al. (1998), von Huene et al. (2000), Husen et al. (2002), Hühnerbach et al. (2005) y Sak

Esta región sobre el monte submarino se caracteriza por patrones complejos de fallas normales y deslizamientos (Sak et al., 2009; Hühnerbach et al., 2005), por lo que se esperarían fallas normales producidas por un monte submarino en subducción, debido al colapso del material de la placa superior (Dominguez et al., 1998).

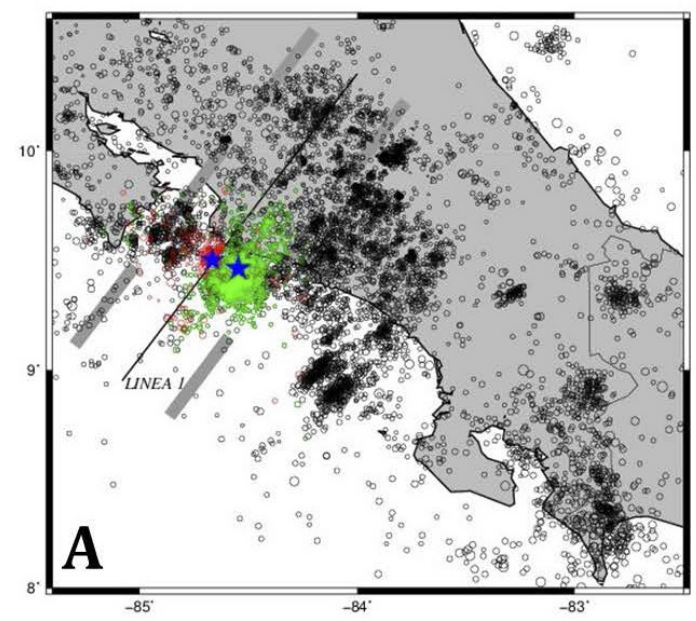
et al. (2009).

\section{Conclusiones}

El sismo de Herradura, 21:51:08 hora UTC, ubicado en el Pacífico Central de Costa Rica, corresponde a una falla inversa, con hipocentro a $18 \mathrm{~km}$ de profundidad, su centroide se encuentra debajo del epicentro a $25 \mathrm{~km}$ de profundidad y +1,3 segundos después del tiempo de origen, con deslizamiento en forma circular, distribución uniforme y máximos deslizamientos de $0.1 \mathrm{~m}$ y magnitud Mw 5.9. Sismos con este tipo de mecanismo focal están asociados a la interfaz CO-CA y asociados con la subducción de la placa de Cocos bajo la placa Caribe. La disminución de la sismicidad que se observa debajo de la interfaz podría estar relacionado a un monte submarino cortado en la placa subducida.

La ubicación del centroide y de las réplicas del sismo de Herradura muestran que los hipocentros se encuentran concentrados en un área pequeña y que no existe rompimiento a lo largo de la inmersión de la placa de Cocos bajo la placa Caribe; lo mismo ocurrió con el sismo de Esterillos, a las 02:28:20.3 hora UTC, con magnitud Mw 6.4 con centroide por debajo del epicentro y máximos deslizamientos de $0.6 \mathrm{~m}$ y con concentración de

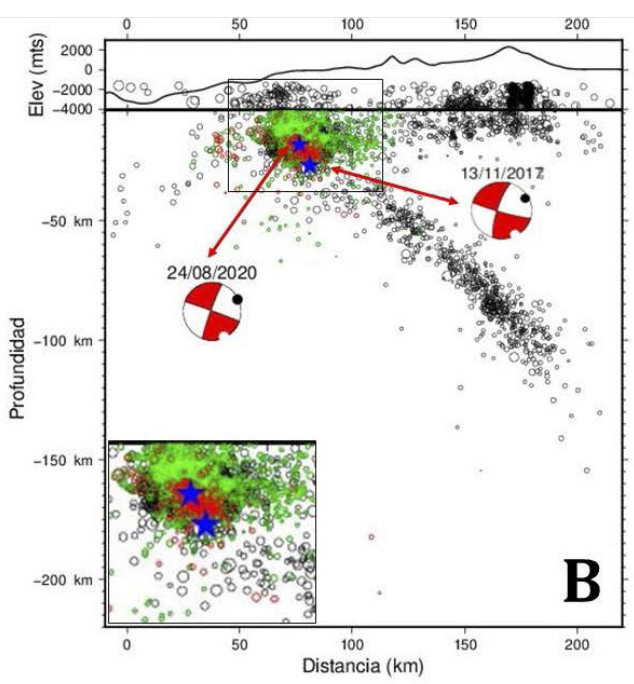

Figura 6 Epicentros de sismos registrados por la red sísmica del OVSICORI-UNA, entre 2010 hasta septiembre del 2020 representados por círculos negros, verdes y rojos, los dos últimos indican epicentros para los primeros 10 días de réplicas después de ocurrido el sismo de Esterillos y Herradura, respectivamente, B) Proyección vertical de los sismos que se encuentran a una distancia máxima de 30 km (líneas grises gruesas en mapa), a ambos lados de la línea 1 y las proyecciones de sus MF en la línea 1 , se muestra el eje $\mathrm{P}$ y $\mathrm{T}$, con círculos blancos y negros, respectivamente. Con estrellas azules se indican las localizaciones de los sismos de Herradura y Esterillos. Se presenta una ampliación en la esquina inferior izquierda de los eventos principales. 
réplicas hacia la superficie y en zonas con cambio de esfuerzos de Coulomb negativos.

La acumulación de esfuerzos estáticos como lo muestra el cambio de esfuerzos de Coulomb se da en las fronteras superior e inferior de la falla en la zona de interfaz sismogénica entre la placa de Cocos y la placa Caribe, zona que se caracteriza por haber subducido montes submarinos que han sido generadores de sismos fuertes en el pasado. La distribución de deslizamientos muestra concentración alrededor del centroide y sin deslizamiento hacia la parte inferior de la interfaz sismogénica; por lo que la distribución de esfuerzos y deslizamiento en la falla producto de estos sismos puede indicar que la parte inferior de la zona sismogénica en esta parte del Pacífico Central de Costa Rica
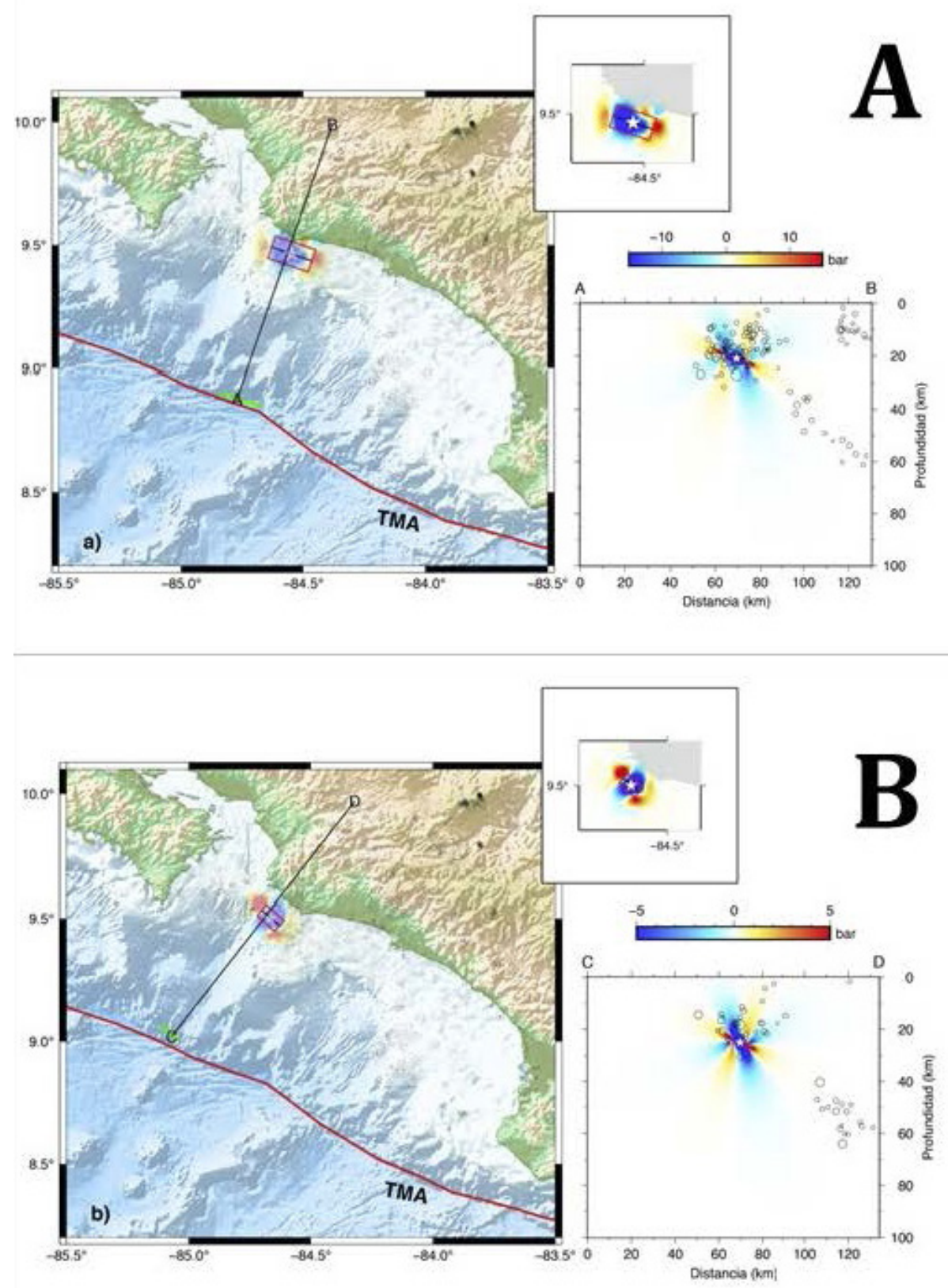

Figura 7 A) Cambios de esfuerzos estáticos de Coulomb debido al sismo del Esterillos (parámetros de falla 289॰/17\%/85, Mo=4.35e+18 $\mathrm{Nm}, \mathrm{Mw} 6.4$, profundidad $21 \mathrm{~km}$, epicentro indicado por estrella blanca, B) Cambios de esfuerzos estáticos de Coulomb debido al sismo de Herradura (parámetros de falla $11 \% / 20 \circ / 98, M o=9.719 \mathrm{e}+17, \mathrm{Mw} 5.9$, profundidad $25 \mathrm{~km}$ ), epicentro indicado por estrella blanca, se asume un coeficiente de fricción de 0.4 . La barra de colores indica los cambios de esfuerzos de Coulomb en bares y con círculos negros se muestran algunos de las localizaciones del catálogo sísmico del OVSICORI-UNA. Una línea de color rojo muestra la TMA y en negro y verde se indica la falla en profundidad y la proyección en superficie, un cuadrado en rojo indica la dimensión de falla. 


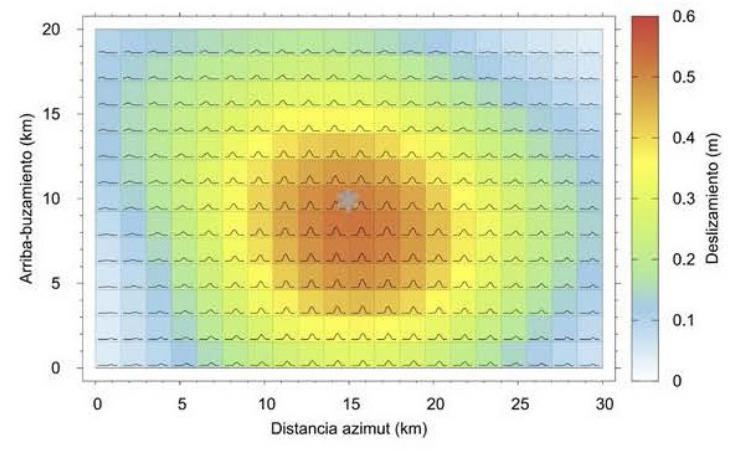

A

Figura 8 Esterillos, el centroide proyectado en el plano de falla (no se usa en la inversión). La distancia es a lo largo del azimut. tiene alto potencial sísmico. Es posible que un monte submarino en la zona esté actuando como barrera que impide la propagación de la ruptura a lo largo de la zona de acople CO-CA.

En la proyección de la sismicidad por profundidad se observa que los sismos en la zona de contacto de las cortezas CO-CA se encuentran concentradas en la zona más superficial quedando la zona inferior de la interfaz con capacidad de romper con otro sismo fuerte o por el contrario actuar como barrera que impida el rompimiento hacia debajo de la zona sismogénica.

La magnitud del sismo de Herradura fue fuerte (Ml 6.1; Mw 5.9), sin embargo, al no ser un sismo superficial y al romper en la TMA y concentrar sus réplicas en esta zona, no generó daños en ciudades y poblados cercanos al epicentro, tal como la ciudad de Jacó (ciudad más próxima). Las intensidades instrumentales en la escala de Mercalli Modificada (MMI) máximas de VI y aceleraciones pico de $\sim 12 \%$ g en la estación más cercana al epicentro, provocó caída de objetos y pánico en las personas en el área de mayor aceleración del suelo, sin embargo, no se reportaron daños a estructuras o pérdidas de vidas.

\section{Agradecimientos}

Agradecemos al editor en jefe Dr. Antoni Camprubí y a los dos revisores anónimos por sus

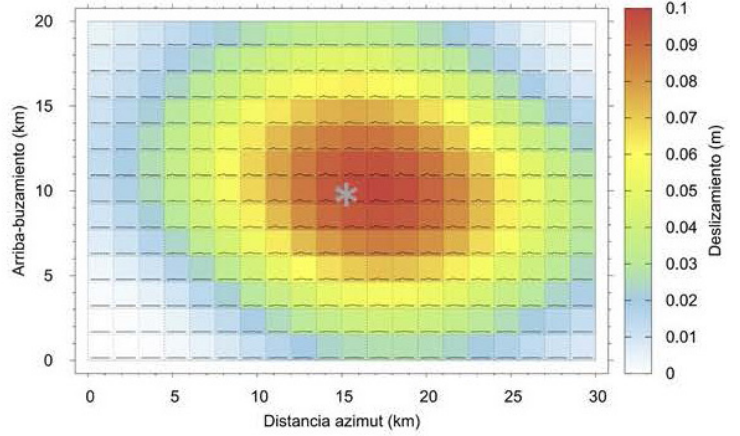

B 
Bilek, S., Schwartz, Y., Heather, R., DeShon, H., 2003, Control of seafloor roughness on earthquake rupture behavior: Geology, 31 (5), 455-458. https://doi.org/10.1130/00917613(2003)031<0455:COSROE > 2.0. $\mathrm{CO} ; 2$

Boulder Real Time Technologies (BRTT), 2010, Software Antelope, Boulder, Colorado. www. brtt.com

Bratt, S.R., Bache, T.C., 1988, Location Estimation using regional array data: Bulletin of the Seismological Society of America, 78, 780-798.

Dominguez, S., Lallemand, S. E., Malavieille, J., von Huene, R, 1998, Upper plate deformation associated with seamount subduction: Tectonophysics, 293(3-4), 207-224. https:// doi.org/10.1016/S0040-1951(98)00086-9

Dziewonski, A., Chou T., Woodhouse, J., 1981, Determination of earthquake source parameters from waveform data for studies of global and regional seismicity: Journal of Geophysical Research: Solid Earth,86, 2825-2852. https://doi:10.1029/ JB086iB04p02825

Ekström, G., Nettles, A., Dziewonski, M., 2012, The global CMT project 2004-2010: Centroid-moment tensors for 13,017 earthquakes: Physics of the Earth and Planetary Interiors, 200-201, 1-9. https:// doi:10.1016/j.pepi.2012.04.002

Fan, G., Pavlis, G., Tuncay, K., 2006, GCLGRID: A three-dimensional geographical curvilinear grid library for computational seismology: Computers in Geosciences, 32, 371-381. https://doi.org/10.1016/j. cageo.2005.07.001

Fisher, D., Gardner, T., Marshall, J., Sak, P., Protti, M., 1998, Effect of subducting seafloor roughness on fore-arc kinematics, Pacific coast, Costa Rica: Geology, 26(5), 467-470. https://doi.org/10.1130/0091$7613(1998) 026<0467$ :EOSSFR>2.3 . $\mathrm{CO} ; 2$

Gallovic, F., Imperatori, W., Mai, P. M., 2014, Effects of three-dimensional crustal structure and smoothing constraint on earthquake slip inversions: case study of the Mw6.3 2009 L'Aquila earthquake: Journal of Geophysical Research: Solid Earth, 120, 428-449, https:// doi.org/10.1002/2014JB011650

Gallovic, F., Zahradník, J., 2011, Toward understanding slip-inversion uncertainty and artifacts 2: Singular value analysis: Journal of Geophysical Research, 116, 1-12. https:// doi.org/10.1029/2010JB007814

Gardner, T., Verdonck, D., Pinter, N., Slingerland, R., Furlong, K., Bullard, T., Wells, S., 1992, Quaternary uplift astride the aseismic Cocos Ridge, Pacific coast, Costa Rica: GSA Bulletin, 104 (2), 219-232. https://doi.org/10.1130/00167606(1992)104<0219:QUATAC>2.3. $\mathrm{CO} ; 2$

Guendel, F., 1986, Seismotectonics of Costa Rica: An analytical view of the southern terminus of the Middle America Trench: Universidad de California, USA, Tesis doctoral.

Global Catalogue, 2020, Global CMT Catalogue. https://www.globalcmt.org/CMTsearch. html

Hauff F., Hoernle K., Schmincke H.U., Werner R., 1997, A Mid Cretaceous origin for the Galapagos hotspot: volcanological, petrological and geochemical evidence from Costa Rican oceanic crustal segments: International Journal of Earth Sciences, 86, 141-155. https://doi.org/10.1007/ PL00009938

Hühnerbach, V., Masson, D. G., Bohrmann, G., Bull, J. M., Weinrebe, W, 2005, Deformation and submarine landsliding caused by seamount subduction beneath the Costa Rica continental margin-new insights from highresolution sidescan sonar data: Geological Society, London, 244(1), 195-205. https:// doi.org/10.1144/GSL.SP.2005.244.01.12

Husen, S., Kissling, E., Quintero, R., 2002, Tomographic evidence for a subducted seamount beneath the Gulf of Nicoya, Costa Rica: The cause of the $1990 \mathrm{Mw}=$ 7.0 Gulf of Nicoya earthquake: Geophysical 
Research Letters, 29, 79-1-79-4. https:// doi:10.1029/2001GL014045

Hutton, K., Boore, D., 1987, The ML scale in southern California: Bulletin of the Seismological Society of America, 77 (6), 2074-2094.

Hoernle, K., Hauff, F., 2007, Oceanic igneous complexes in Central America, en Bundschuh, J., Alvarado, G. (Eds.), Central America: Geology, Resources and Hazards. London: GRG Press, 523-548. https://doi. org/10.1201/9780203947043

International Seismological Center, 2020, Summary of the Bulletin of the ISG. http:/ / www.isc.ac.uk

Kennett, B., Engdahl E., 1991, Traveltimes for global earthquake location and phase identification: Geophysical Journal International, 122, 429-465. https://doi. org/10.1111/j.1365-246X.1991.tb06724.x

Klein, F., 1984, User's guide to Hypoinverse, a program for Vax and Pc350 computers to solve for earthquake locations: U. S. Geological Survey, Open File Report 84-000. https://doi.org/10.3133/ofr89314

Kreemer, C., Blewitt, G., Klein, E., 2014, A geodetic plate motion and Global Strain Rate Model: Geochemistry, Geophysics, Geosystems, 15, 3849-3889. https://doi. org/10.1002/2014GC005407

Kolarsky, R., Mann, P., 1995, Structure and neotectonics of an oblique-subduction margin, southwestern Panama, en Mann, P. (ed.), Geologic and tectonic development of the Caribbean Plate Boundary in Southern Central America: GSA, Special Papers, 131-157. https://doi.org/10.1130/ SPE295-p131

Lienert, B. R., Haskov, J., 1995, A computer program for locating earthquakes both locally and globally: Seismological Research Letters, 66, 26 - 36. https://doi.org/10.1785/ gssrl.66.5.26

Lin, J., R.S. Stein., 2004, Stress triggering in thrust and subduction earthquakes, and stress interaction between the southern San Andreas and nearby thrust and strike-slip faults: Journal of Geophysical Research, 109, 1-19. https://doi:10.1029/2003JB00260

Marshall, J., Fisher, D., Gardner, T., 2000, Central Costa Rica deformed belt: Kinematics of diffuse faulting across the western Panama Block: Tectonics 19(3), 468-492. https://doi. org/10.1029/1999TC001136

Mescua, J. F., Porras, H., Durán, P., Giambiagi, L., de Moor, M., Cascante, M., Poblete, F., 2017, Middle to late Miocene contractional deformation in Costa Rica triggered by plate geodynamics: Tectonics, 36(12), 2936-2949. https://doi.org/10.1002/2017TC004626

Richter, C. F., 1935, An instrumental earthquake magnitude scale: Bulletin of the Seismological Society of America, 25,1-32.

OVSICORI-UNA, 2020, Catálogo sísmico en línea. http://www.ovsicori.una.ac.cr/index. $\mathrm{php} /$ localizacion-automatica.

OVSICORI-UNA, 24 de agosto de 2020, Reporte de sismo día: 2020-08-24, [Publicación de estado]. Facebook. https://www.facebook. com/OVSICORI/photos/a.120485684655 365/3126011160769454/?type $=3$

Protti, M., Güendel, F., McNally, K., 1994, The geometry of the Wadati-Benioff zone under southern Central America and its tectonic significance: results from a highresolution local seismographic network: Physics of the Earth and Planetary Interiors, 84, 271-287. https://doi. org/10.1016/0031-9201(94)90046-9

Protti, M., Güendel, F., McNally, K., 1995a, Correlation between the age of the subducting Cocos Plate and the geometry of the WadatiBenioff zone under Nicaragua and Costa Rica: Geologic and Tectonic Development of the Caribbean Plate Boundary in Southern Central America, 309-326. https://doi. org/10.1130/SPE295-p309

Protti, M., McNally, K., Pacheco, J., González, V., Montero, C., Segura, J., Brenes, J., Barboza, V., Malavassi, E., Güendel, F., Simila, G., 
Rojas, D., Velasco, A., Mata, A., Schillinger, W., 1995b, The March 25, 1990 (Mw = 7.0, $\mathrm{ML}=6.8$ ), earthquake at the entrance of the Nicoya Gulf, Costa Rica: Its prior activity, foreshocks, aftershocks, and triggered seismicity: Journal of Geophysical Research Solid Earth, 100 (B10), 20345- 20358. https://doi.org/10.1029/94JB03099

Proyecto Global Centroide Momento Tensor (GCMT), 2020, Catalogo en línea. https:// www.globalcmt.org/

Quintero, R., Kissling, E., 2001, An improved $\mathrm{P}$-wave velocity reference model for Costa Rica: Geofísica Internacional, (40) 1, 3-19.

Quintero, R., Zahradník, J., Sokos, E., 2014, Nearregional CMT and multiple-point source solution of the September 5, 2012, Nicoya, Costa Rica Mw 7.6 (GCMT) earthquake: Journal of South American Earth Sciences, 55, 155-165. https://doi.org/10.1016/j. jsames.2014.07.009

Sak, P. B., Fisher, D. M., Gardner, T. W., Marshall, J. S., LaFemina, P. C., 2009, Rough crust subduction, forearc kinematics, and Quaternary uplift rates, Costa Rican segment of the Middle American Trench: Geological Society of America Bulletin, 121(7-8), 9921012. https://doi.org/10.1130/B26237.1

Sallares, V., Charvis, P., Flueh, E.R., Bialas, J., 2003, Seismic structure of Cocos and Malpelo Volcanic Ridges and implications for hot spot-ridge interaction: Journal of Geophysical Research, 108(B12), 2564, https://doi:10.1029/2003JB002431

Sallares, V., Charvis, P., Flueh, E. R., Bialas, J., Salieri S, 2005, Seismic structure of the Carnegie ridge and the nature of the Galapagos hotspot: Geophysical Journal International, 161(3), 763-788. https://doi. org/10.1111/j.1365-246X.2005.02592.x

Servicio Geológico de Estados Unidos (USGS), 2020, M 6.0 - 4 km S of Jacó, Costa Rica. Nota técnica.

Sokos, E., Zahradník, J., 2008, ISOLA a Fortran code and a Matlab GUI to perform multiple-point source inversion of seismic data: Computers and Geosciences, 34, 967-977. https://doi.org/10.1016/j. cageo.2007.07.005

Sokos, E., Zahradník, J., 2013, Evaluating centroid_moment tensor uncertainty in the new version of ISOLA software: Seismological Research Letters, 84 (4), 656665. https://doi.org/10.1785/0220130002

Snoke, J., Munsey, J., Teague, A., Bollinger, G., 1984, A program for focal mechanism determination by combined use of polarity and SV-P amplitude ratio data: Earthquake notes, 55 .

Toda, S., R. S. Stein., K. Richards-Dinger and S. Bozkurt, 2005, Forecasting the evolution of seismicity in southern California: Animations built on earthquake stress transfer: Journal of Geophysical Research B: Solid Earth, 110, 1-17. https://doi. org/10.1029/2004JB003415

Tsuboi, S., Abe, K., Takano, K., Yamanaka, Y., 1995, Rapid Determination of Mw from Broadband $\mathrm{P}$ waveforms: Bulletin of the Seismological Society of America, 85 (2), 606-613.

von Huene, R., Ranero, C.R., Weinrebe, W., Hinz, K., 2000, Quaternary convergent margin tectonics of Costa Rica, segmentation of the Cocos Plate, and Central American volcanism: Tectonics, 19(2), 314-334. https://doi.org/10.1029/1999TC001143

von Huene, R., Bialas, J., Flueh, E., Cropp, B., Csernok, T., Fabel, E., Hoffman, J., Emeis, K., Holler, P., Jeschke, G., Leandro, C. M., Perez, I., Chavarria, J., Florez, A., Escobedo, D., Leon, R., Barrios, O., 1995, Morphotectonics of the Pacific convergent margin of Costa Rica, en Mann, P. (Ed.) Geologic and Tectonic Development of the Caribbean Plate Boundary in Southern Central America: Geological Society of America, Special Paper, 295, 291-307. https://doi.org/10.1130/SPE295-p291

Wald, D., Worder, B., Quitoriano, V., Pankow, K., 2005, ShakeMap manual: technical manual, user's guide, and software guide. U.S. 
Geological Survey, Techniques and Methods 12-A1, 132.

Walther, C.H., Bialas, J., Flueh, E.R., 2000, Crustal structure of the Cocos Ridge off Costa Rica. Preliminary results from a seismic wide-angle experiment, EOS: American Geophysical Union Fall Meet. Suppl., 81, Abstract T11A-03.

Walther, C., 2003, The crustal structure of the Cocos ridge off Costa Rica: Journal of Geophysical Research Solid Earth, 108(B3), 2136. https:// doi.org/10.1029/2001JB000888

Walther, C., Flueh, E., 2002, Remnant of the ancient Farallon Plate breakup: a lowvelocity body in the lower oceanic crust of Nicoya Peninsula, Costa Rica-evidence from wide-angle seismic: Geophysical Research Letters, 29, 1939. https:// doi:10.1029/2002GL015026
Watts, B., Koppers, A., Robinson, D., 2010, Seamount subduction and earthquakes: Oceanography, 23(1),166-173. https://doi. org/10.5670/oceanog.2010.68

Werner, R., Hoernle., K., Van Den Bogaard P., Ranero, C., von Huene, R., Korich, D., 1999, Drowned 14-m.y.-old Galapagos archipelago off the coast of Costa Rica: implications for tectonic and evolutionary models: Geology, 27 (6), 499-502. https://doi.org/10.1130/00917613(1999)027<0499:DMYOGP>2.3 . $\mathrm{CO} ; 2$

Wilson D.S., Teagle D.A., Acton G.D., 2003, First results from hole $1256 \mathrm{D}-\mathrm{a}$ new ocean-crust reference hole drilled in fastspread crust during ODP Leg 206, Eos Transactions American Geophysical Union, 84(46),U12A-02. 